\title{
Nonintrusive eye gaze tracking using a single eye image
}

\begin{abstract}
This paper proposes an Eye Gaze Tracking (EGT) technique using a single eye image that can be easily calibrated and mapped for Human Computer Interaction (HCI). The technique employs both geometric and trigonometric relationships to find a user's Point of Regard, followed by calculating user-dependent variables for final mapping onto a user interface (UI). Experimental results show acceptable accuracy with minimal focal errors.
\end{abstract}

Keyword: Computer vision; Eye gaze tracking (EGT); Human computer interaction (HCI) 